\title{
APERÇU SUR LE PEUPLEMENT ENTOMOLOGIQUE DES EAUX PYRÉNÉENNES (1)
}

\author{
par Henri BERTRAND \\ Docteur es Sciences.
}

Il ne peut être question, à l'heure actuelle, que de donner un aperçu du peuplement entomologique des eaux pyrénéennes destiné à en montrer tout l'intérêt.

En effet, la faune entomologique des eaux douces des Pyrénées n'a jamais été l'objet d'un travail d'ensemble qui nécessiterait le concours de nombreux spécialistes. Toutefois, nous disposons déjà de matériaux assez importants sur quelques groupes : Trichoptères et Plécoptères, surtout grâce aux recherches de Mosely et DESPAX; quelques indications nous ont été données aussi par Eaton, Lacrorx, Navas. D’ailleurs quelques points de la chaîne seuls ont été plus ou moins explorés et il y aurait certainement encore beaucoup à faire pour dresser un inventaire de la faune pyrénéenne; aussi mon exposé sera-t-il surtout basé sur mes propres observations et, par conséquent, bien incomplet...

Mais, avant de parler des insectes des eaux pyrénéennes, il ne paraîtra pas inutile, je le pense, de procéder à un bref rappel de la nature, de la structure et de l'histoire des Pyrénées; en dernière analyse, constitution du sol, évolution du relief et du climat, vicissitudes du lointain passé géologique encore, sont à l'origine des caractéristiques des "biotopes" aquatiques, partant de leur faune comme de leur flore.

Les Pyrénées sont de très vieilles montagnes et, par là comme par bien d'autres traits, elles diffèrent des Alpes; les flots des océans anciens recouvraient encore l'emplacement de ces dernières, que déjà émergeaient des terres pyrénéennes, déjà colonisées par plantes et animaux et ou vivaient, bien entendu, des insectes. Mais ces premières Pyrénées, chaîne " hercynienne " qui étaient sans doute déjà de hautes montagnes, ont été rapidement usées par l'érosion et réduites à ce que les géographes appellent des " pénéplaines ", de hauts plateaux comparables à notre Massif Central. Aujourd'hui, il faut nous contenter de les évoquer en consultant la carte géologique où sont figurés les anticlinaux dévoniens ou siluriens et les grands synclinaux carbonifères, par exemple dans les régions de Cauterets et Barèges. Comme on le sait, ces anciens

(1) Extraits d'une conférence faite à la Société Centrale d'Aquiculture et de Pêche. 
bouleversements du sol ont été accompagnés de phénomènes éruptifs : ainsi des roches éruptives de profondeur, des granites, ont échauffé, " digéré " ces dépôts carbonifères ; plus résistantes, elles forment encore la plupart des grands sommets pyrénéens et aux points de contact par exemple entre Cauterets et la Raillière - chacun peut voir ces vieux schistes recuits, les "injections" de granit, les blocs de schistes ou de grès pris en "sandwiches" dans la roche granitique, cependant qu'apparaissent des minéraux de métamorphisme tels les grenats... Chacun sait aussi que la noire silhouette en racine de molaire du Pic du Midi d'Ossau, est le vestige d'un ancien volcan à andésite. Et les dépôts rouges détritiques des Nogueras en Espagne, ou ceux des environs des Forges d'Abel dans la haute vallée d'Aspe, témoignent du démantèlement de la chaîne. La mer empiète sur la terre et du sel se dépose dans les lagunes

Mouvements locaux ou généraux d'émersion et de submersion se poursuivent au cours des âges et nous n'entrerons pas ici dans leur détail ; retenons seulement que, sans qu'il y ait eu un nouveau plissement, une srande partie de la pénéplaine est émergée au Jurassique moyen. Au contraire, vers le Crétacé moyen, débute une vaste offensive de la mer; c'est alors que se déposent ces puissantes assises qui ont donné notamment les paysages grandioses du cirque de Gavarnie et, sur le versant espagnol, ceux d'Arazas et du Cotatuero et, en haut du Balaïtous, il reste un bloc de calcaire, vestige de cette mer.

$\mathrm{Au}$ cours de cette lutte entre la terre et la mer, s'établissent des relations variées en étendue et en durée entre la région pyrénéenne et les autres terres et continents émergés et l'on sait que la composition même des faunes entomologiques, particulièrement des faunes cavernicoles, peut s'expliquer par ces vicissitudes, quand elles ne viennent pas, mème, jusqu'à les préciser. Notons surtout que les principaux dépôts géologiques pyrénéens sont constitués par des roches anciennes, résistantes, souvent métamorphisées : grès compacts, schistes durs, calcaires marmorisés; à ce point de vue, les Pyrénées diffèrent des Alpes et cela aussi joue un rôle dans l'aspect des eaux et du paysage pyrénéen.

C'est vers la fin des temps tertiaires que naissent les Pyrénées actuelles. Alors, de formidables poussées façonnent la chaîne, non seulement les couches se dressent, mais les plis se cabrent, glissent les uns sur les autres, comme des piles de livres sur une table, et les géologues distinguent ainsi une série de "nappes " pyrénéennes ; parfois, des morceaux du support ancien sont emportés en avant et plusieurs des " avantmonts " de la chaîne, notamment vers l'Est, sont des fragments détachés du socle axial. Dans la vallée d'Ossau, par exemple, encore dans la région de Gavarnie, on peut voir ces étranges superpositions de terrains, faisant alterner roches anciennes et couches secondaires, et mème des couches tertiaires, dépôts de la mer d'Espagne, culminent à 3.353 mètres, au Mont-Perdu, où Ramond découvrit des fossiles marins.

Mais l'érosion s'attaque à la nouvelle chaîne, comme à l'ancienne; déjà se creusent de profondes vallées; il y a un golfe vers l'Adour, des lacs en Aquitaine, et des effondrements isolent la pointe orientale de la chaîne entre les golfes du Roussillon et de l'Ampurdan; alors, comme 


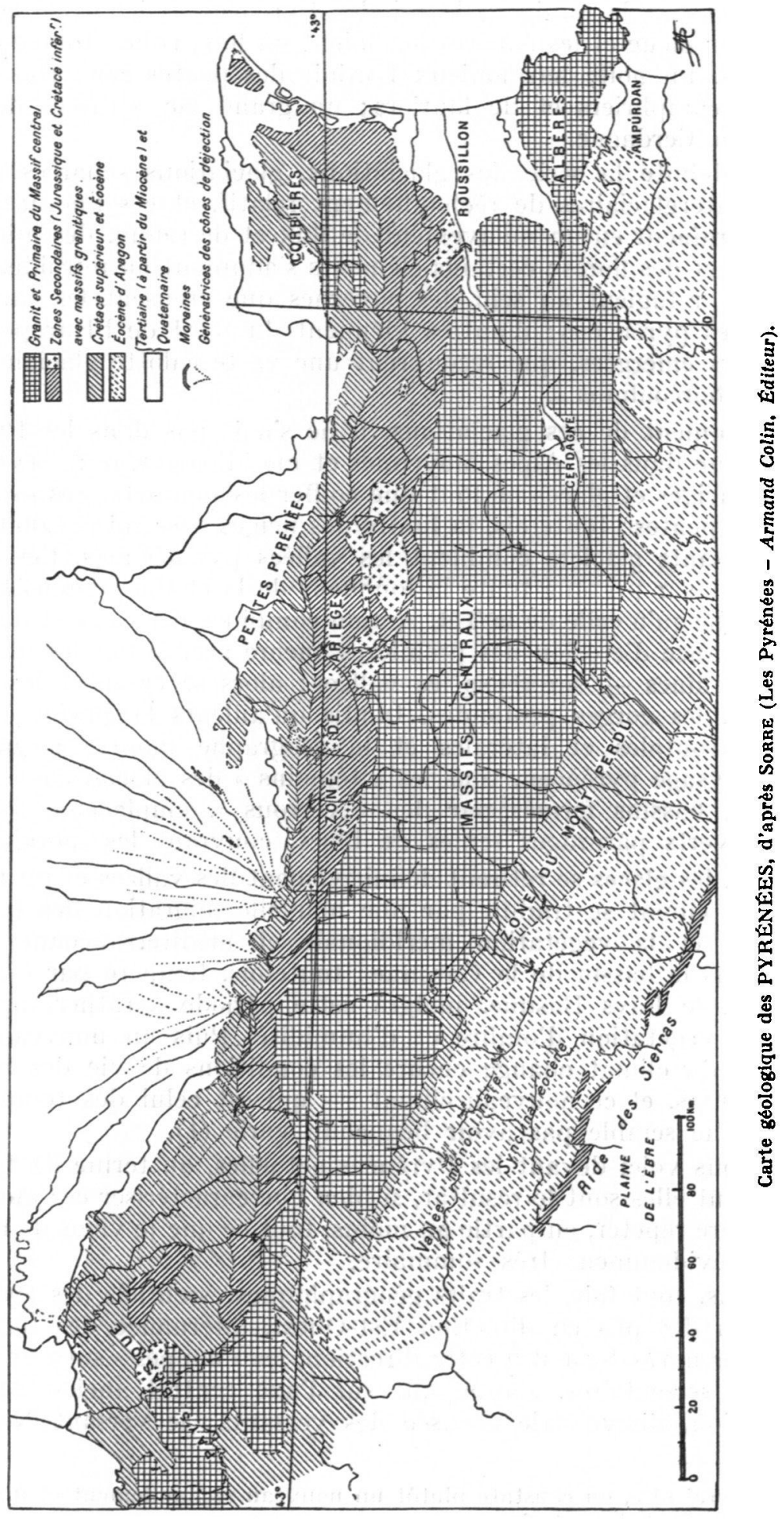


l'écrit Gaussen, le Canigou, le roi des Pyrénées, baignait directement dans la mer bleue et les Albères formaient un long ruban tortueux entre deux mers, un golfe harmonieux bordait des pentes couvertes de palmiers, de camphriers et de lauriers; un grand lac, entouré de forêts occupait la Cerdagne.

Puis débute la série des glaciations, glaciations séparées par des périodes interglaciaires de réchauffement relatif, et ces fluctuations du climat entraînent en un mouvement de flux et de reflux, des migrations des faunes et des flores ; certains éléments s'adaptent au refroidissement, d'autres se réfugient au sein des cavernes que creusent les eaux dans les calcaires pyrénéens; d'autres viennent du nord de l'Europe et des Alpes, alors émergées, que recouvrent une vaste calotte glaciaire, et ce sont les "boréoalpins".

Au moment de ces glaciations, il ne s'agit pas dans les Pyrénées, pas plus que dans les Alpes moyennes et méridionales, d'un revêtement continu : neiges et glaces ne font que coiffer les sommets, des forêts garnissent les pentes; toutefois, la limite des neiges descend à 1.000 mètres et de puissants glaciers comblent les vallées pyrénéennes. Ces glaciers s'étendaient sur presque toute la longueur de la chaîne, depuis la vallée du Saison jusqu'à celle de la Têt. Action combinée des eaux et des glaces - la puissance d'érosion des premières encore accrue par les matériaux solides entraînés - surcreuse les vallées; alors se creusent les cirques grandioses, les gorges profondes, tandis qu'ailleurs le glacier étale ses moraines frontales et latérales et le géographe montre aujourd'hui, dans le paysage pyrénéen, tous les "témoins" des glaciations : vallons suspendus, bassins fermés par des "verrous ", emplacement de lacs disparus, verts pâturages semés de blocs, couvrant les moraines.

Enfin, les glaces se retirent définitivement des vallées et un réchauffement du climat se traduit par une nouvelle migration des faunes et des flores, et la végétation méridionale et méditerranéenne pénètre jusque dans le bassin de la Garonne; toutefois, tempéré par l'influence atlantique, le Pays Basque, malgré cette période xérothermique conserve une végétation d'ombre et d'humidité. Puis un nouveau refroidissement du climat change encore les conditions de vie des animaux et des plantes, et ce nouveau climat - qui est celui des temps historiques - ne semble pas avoir beaucoup varié (1).

Et nous voici devant les Pyrénées actuelles, au terme de tant d'épisodes dont elles sont le résultat, si bien que caractériser ces montagnes serait encore répéter, en partie au moins, tout ce que je viens de retracer, de façon évidemment très sommaire.

Notons, toutefois, les traits géographiques essentiels des Pyrénées : orientation des plis en direction Est-Ouest, alternance des formations géologiques normalement à cette direction, les " nappes " surtout formées de roches secondaires, s'appuyant sur l'axe central ancien découvert par l'érosion, dissymétrie accusée des versants; le versant Nord plus

(1) A vrai dire, on constate plutốt un nouveau réchauffement et une régression nouvelle des neiges et glaces, 
abrupt, et donc moins ètendu, le versant Sud doucement incliné jusqu'ì la zone des sierras, contraste frappant des climats et aussi, dans une certaine mesure, du sol entre ces mêmes versants : versant espagnol à températures extrêmes, roches calcaires et végétation méridionale, versant français humide et verdoyant, contrastes atténués cependant aux deux extrémités de la chaîne : Catalogne orientale d'une part, Pays Basque de l'autre; dominance enfin sur le versant Nord et surtout vers l'Ouest, des grandes vallées transversales sur les vallées longitudinales souvent largement ouvertes du versant Sud et de la région orientale.

Eaux pyrénéennes... ces mots, chez ceux qui connaissent les Pyrénées, évoquent, j'en suis sûr, la double image du "gave " et de ses cascades dans un site boisé, et du lac bleu, scintillant au soleil, dans le désert de pierrailles. Torrents et lacs, à la fois charme et richesse des Pyrénées.

Les cours d'eau pyrénéens, comme le remarque Sorre, ne sont jamais " des collecteurs comparables aux rivières alpestres"; le "type du cours d'eau pyrénéen est le torrent dévalant dans un court et profond sillon, parallèlement à l'axe de la chaîne ". Aussi les crues sont elles violentes, mais d'écoulement rapide et de courte durée... Ajoutons que si " chez ces cours d'eaux la pente est rapide, il est important de noter qu'elle est souvent discontinue, les gorges profondes se trouvant généralement au pied de la montagne, l'encaissement devenant de moins en moins accusé dans les cours moyen et supérieur ", comme l'indique Gavrier qui cite l'exemple très net fourni par le gave d'Ossau, avec, succédant aux gorges du Hourat, les larges vallées de Brousset et de Bious, les faibles ondulations des cirques d'Anapéou et d'Ayous. Il faut dire aussi que les " accidents " résultent essentiellement de la nature des roches traversées; ainsi voyons-nous ces entailles étroites dans les couches schisteuses, ces défilés à la traversée des bancs de calcaire secondaires et primaires - dans la valée d'Aspe par exemple.

Bien entendu, le torrent pyrénéen n'est point d'un type invariable ; d'ailleurs le régime des eaux varie avec le climat, l'importance, l'époque des précipitations : ainsi, le volume maximum de débit est atteint au printemps dans les Pyrénées méditerranéennes, en hiver dans les Pyrénées centrales, tandis qu'il y a deux maxima dans les Pyrénées atlantiques. Du point de vue plus proprement hydrobiologique, signalons que notre collègue $\mathrm{M}$. P. Grenier, à l'occasion de ses belles recherches sur la biologie des Simulies, a fait de nombreuses observations sur le gave de Cauterets, notant les vitesses de courant, le $P h$, les oscillations journalières de la température et de la teneur en oxygène dissous, montrant qu'au moins les grands gaves pyrénéens dans les parties élevées de leur cours différaient beaucoup des torrents du Massif Central, l'absence pratique de végétation - surtout algale - entraînant, par suite de la disparition de l'action antagoniste de la photosynthèse durant le jour, une chute de l'oxygène aux heures chaudes des journées d'été, les vitesses de courant pouvant atteindre 1 à 2 mètres à la seconde, le $\mathrm{P} h$ restant faible. 
Les lacs. - Les lacs des Pyrénées sont fort nombreux; Gaurier en a répertorié plus de 500 ; leur superficie, généralement médiocre, n'atteint souvent que quelques hectares, parfois moins d'un hectare, les plus grands dépassant trente ou quarante hectares; quant à leur profondeur, inférieure ou égale à quelques dizaines de mètres, elle atteint ailleurs 50 à 50 mètres et rarement 100 mètres. L'étendue de ces lacs est sujette à variations et, comme partout en montagne, ils tendent souvent à se combler, soit par l'alluvionnement, soit par les avalanches, soit par le développement de la végétation; Gaurier cite à ce propos les trois exemples des lacs d'Artouste, de Gaube et d'Estibère. Du point de vue limnologique, les lacs pyrénéens sont mal connus; beaucoup semblent oligotrophes; beaucoup sont privés de végétation phanérogamique, celle-ci se développant toutefois dans pas mal des "étangs" des Pyrénées-Orientales ; les températures varient aussi et en été nous avons ainsi relevé des températures de $15^{\circ}$ et $17^{\circ}$ à la surface et sur les bords, mais quelquefois aussi seulement $13^{\circ}, 7^{\circ}, 8^{\circ}$ ou même $3^{\circ}$ ou $2^{\circ}$ dans ceux encore plus ou moins couverts par les glaces. Mais la caractéristique générale des lacs pyrénéens, et qui offre un intérêt biologique particulier, c'est la haute altitude à laquelle ils se trouvent tous placés. Ainsi, parmi les lacs énumérés par Gaurier, seul le lac de barrage glaciaire de Lourdes est situé au pied de la chaîne, à seulement 421 mètres d'altitude; tous les autres s'échelonnent entre 1.500 et 3.000 mètres, un nombre assez faible se trouvant entre 1.500 et 2.000 mètres, beaucoup entre 2.200 et 2.300 mètres, quelques-uns seulement au-dessus de 2.400 et 2.500 mètres.

Les glaciers. - Bien modestes à côté des glaciers alpins, ils n'existent au reste que dans une partie de la chaîne. La limite inférieure des neiges reste élevée dans les Pyrénées ; variant, bien entendu, indépendamment des facteurs locaux, selon les régions de la chaîne, elle se relève à l'orient jusqu'à 2.700 mètres, au Canigou. Comme il n'y a pas, dans l'ensemble, un intervalle suffisant entre cette limite et les crêtes elles-mêmes, les glaciers ne peuvent se développer comme ceux des Alpes; ce ne sont, comme le dit Sorre, que des " appareils de deuxième ordre, glaciers de cirque (Gavarnie), de plateau (Maladetta, Néouvielle), revêtant au plus l'apparence de glaciers de vallée (Vignemale) ".

Mentionnons encore pour mémoire les eaux souterraines ; on connaît le nombre et l'intérêt des grottes et cavernes pyrénéennes; beaucoup sont parcourues par des eaux, eaux à température diverses, variant de $6^{\circ}$ à $15^{\circ}$, températures comparables à celles d'une bonne partie des eaux de montagnes extérieures. Remarquons seulement qu'au réseau hydrographique souterrain il convient, d'après JEANNEL, d'adjoindre les eaux phréatiques, ce qui, comme le remarque cet auteur, étend singulièrement le domaine souterrain en dehors des massifs calcaires. Les sources, enfin, ne sont pas rares dans la montagne pyrénéenne, sources souvent à basse température.

Mais du point de vue du naturaliste et notamment de l'entomologiste, le domaine des eaux doit être exploré dans le plus infime détail. 
En effet, les plus minimes collections d'eaux ont un grand intérêt et sont d'ailleurs fréquemment les mieux peuplées.

C'est ainsi que les torrents de montagne doivent être suivis jusque dans leur cours supérieur et celui de leurs affluents, particulièrement au niveau de ces hauts pâturages, où ils deviennent de simples ruisseaux, voire des ruisselets; de même, doivent être explorés, à toutes les altitudes, les plus petits ruisseaux. Certains de ces ruisseaux coulent à découvert, d'autres sous le couvert des bois; enfin, assez fréquemment, leurs eaux se chargent de calcaire qu'elles déposent ensuite en abondance, agglomérant les pierres du fond qui se trouve ainsi stabilisé. Bien entendu, les conditions réalisées dans ces milieux diffèrent plus ou moins de celles des grands cours d'eaux; la vitesse du courant se réduit parfois à moins d'un mètre par seconde, la végétation muscinale et surtout algale, atteint souvent un développement notable; enfin, dans certains cas, au moins dans les très petits ruisselets ou dérivations, la température s'élève beaucoup aux heures chaudes de l'été, pouvant atteindre et même dépasser $20^{\circ}$. Une partie de ces eaux proviennent d'ailleurs de rhéocrènes souvent à basse température, comme nous l'avons indiqué déjà et l'on trouve aussi de basses températures dans les eaux de fonte des glaciers et névés, mais ces eaux, dites " glaciaires" n'occupent qu'une place réduite, corrélativement au faible développement, signalé ci-dessus, des

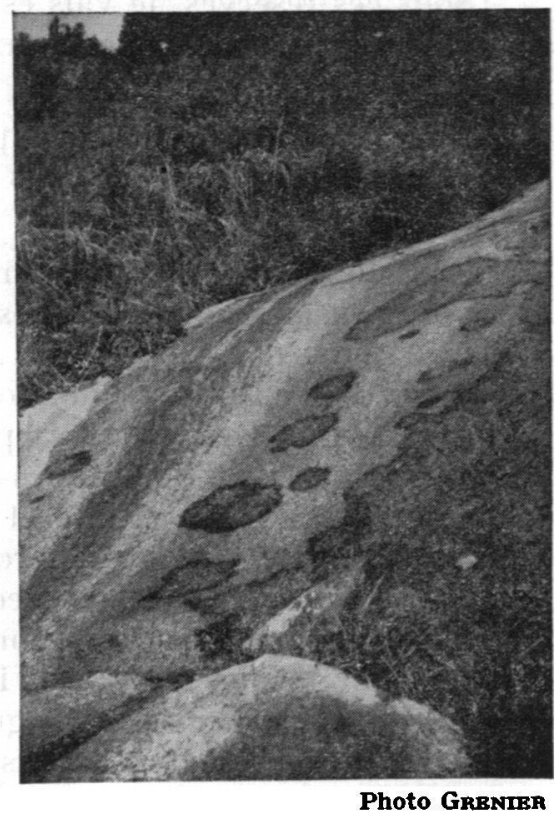

Une surface hygropétrique ruisselante à Cauterets. appareils glaciaires eux-mêmes.

C'est encore aux eaux courantes, plus exactement aux milieux lotiques, qu'il convient de rattacher l'ensemble assez complexe correspondant aux milieux "hygropétriques " sur lesquels Thienemann, le premier, a attiré l'attention. Les milieux hygropétriques les plus typiques, sont, on le sait, constitués par les rochers ruisselants ou suintants, très fréquents dans les Pyrénées, et à toutes les altitudes, mais des " surfaces hygropétriques " se constituent aussi bien aux abords des rhéocrènes, des ruisseaux calcaires, au voisinage des cascades, sur des rochers élevés aux points de fonte des névès, sur les tufs, et parfois jusque dans la saignée d'un simple chemin muletier. Le milieu hygropétrique est caractérisé par l'infime épaisseaur de la couche d'eau et son constant renouvellement, conditions qui, jointes le plus souvent à l'abondant développement d'une végétation algale comprenant parfois des Algues filamenteuses, plus généralement une association de Cyanophycées, Protococcales, Desmidiées et surtout Diatomées, entraînent, malgré une élévation 
de température due à l'insolation fréquente, une suroxygénation remarquable.

De même les eaux stagnantes ne sont pas représentées seulement par les lacs ou laquets, mais par de petites mares, marettes, trous d'eau et fossés qui se développent particulièrement dans les pâturages subalpins souvent aussi marécageux et tourbeux.

Ainsi en montagne, tout autant et même plus qu'en plaine, il existe des passages entre les divers milieux aquatiques, sans parler de leur variation et évolution naturelles.

Sous ces réserves, je vais essayer de montrer sommairement la physionomie générale des principaux peuplements entomologiques des eaux pyrénéennes.

Bien entendu, dans ces peuplements, ce sont ici les éléments lotiques qui dominent et nous commencerons par examiner la faune des gaves.

Sans nous engager au cœur même de la montagne, il suffit d'explorer le lit de l'un quelconque de nos grands gaves pyrénéens, même à assez basse altitude, par exemple entre 500 et 600 mètres, pour observer une faune particulière. Dès que l'on soulève quelques grosses pierres, on découvre ces grosses nymphes pétricoles et lucifuges, bien connues des pêcheurs de Truites : nymphes de Perla (Plécoptères) et nymphes d'Ephémeroptères à tête aplatie et yeux dorsaux de la famille des Ecdyonurides : Epeorus à deux cerques, Ecdyonurus et Rhitrogena à trois cerques, les secondes bien reconnaissables aux lamelles branchiales antérieures et postérieures se prolongeant et s'affrontant sous le ventre comme pour former ventouse. Il y a aussi de plus petites formes : des Nemurides (Protonemura) du groupe des Plécoptères et les Ephémeroptères Baetis, représentés parfois par des Baetis vernus, mais plus généralement par des nymphes à cerque médian court, parfois atrophié 13. gemellus et surtout alpinus, abondants notamment dans les touffes de mousses et, dans le sable, on voit glisser les frêles et souples nymphes i fourreaux alaires parallèles des Leuctra (Plécoptères). Bien entendu, on trouve également des larves de Trichoptères : les unes "nues ", larves des Rhyacophila, Hydropsyche, Polycentropus, Philopotamus et larves à fourreau de fins grains de sables ou de pierrettes des Odontocerum, des Stenophylax. Parfois, sous l'amas fixé de pierrettes, on rencontre les larves nues en prénymphose ou même leur cocon gris ou brun et parcheminé; parfois des larves au moment de la métamorphose groupent leurs fourreaux aciculaires (Leptocerides) ou bien encore le "lestent" d'un petit caillou. Les Diptères ne sont point absents; les plus grosses larves sont les larves grises à atrium étoilé (étoile à six branches) des Tipula, encore des Limnophila à extrémité abdominale renflée, accompagnées des larves des Brachycères : larves fusiformes à faux pieds des Atherix (Rhagionides), larves à bourrelets transverses des Tabanides. Il existe aussi, parmi les petites larves, des larves de Chironomides, particulièrement des Orthocladiinae. Mais l'attention est surtout attirée par les larves et les nymphes des Simulies, parfois en nombre au niveau 


\section{QUELQUES INSEGTES DES EAUX PYRÉNÉENNES}
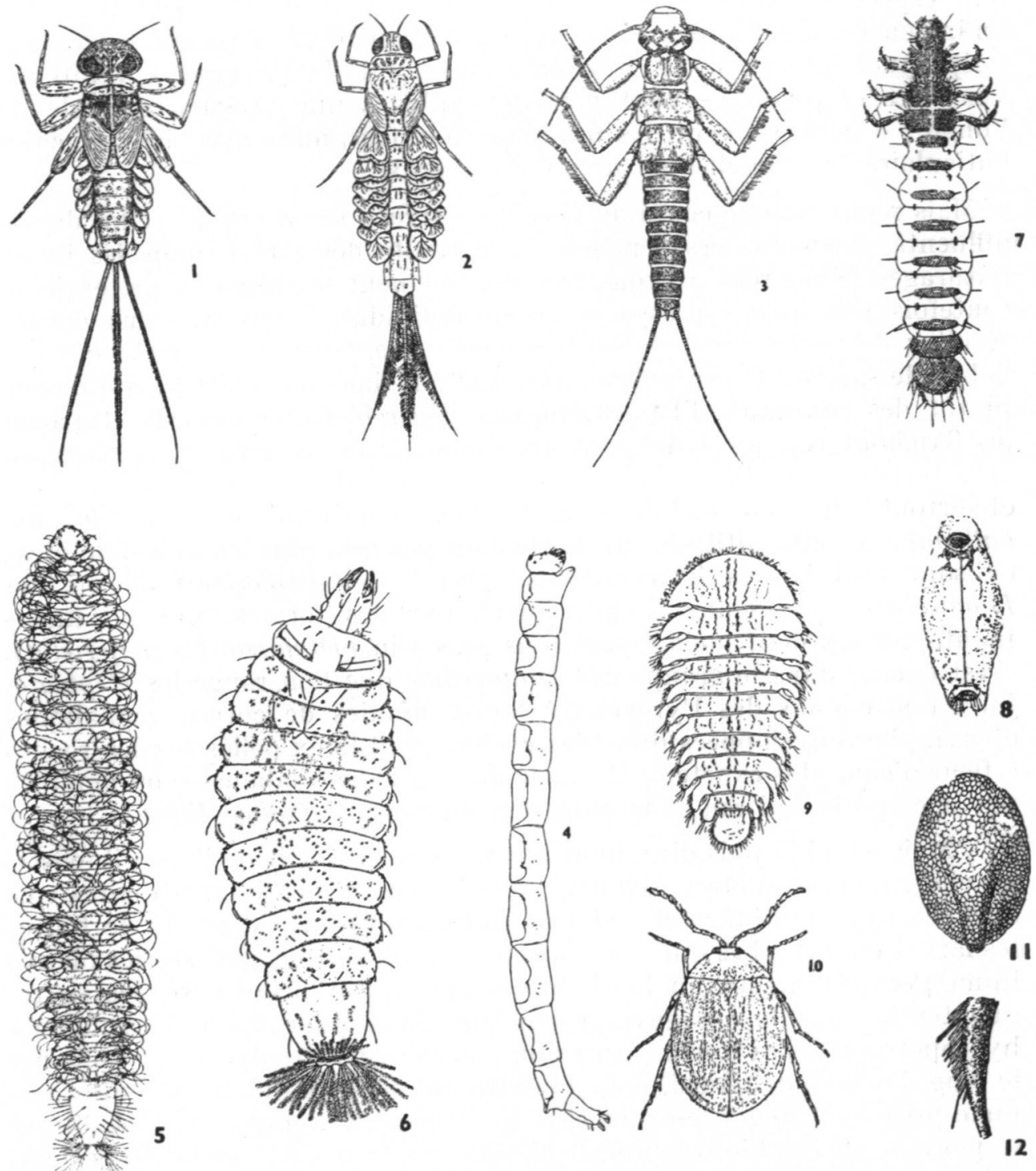

1, larve nymphe de Rhitrogena (Ephéméroptères), torrents et eaux glacialres; 2, larve nymphe de Siphlonurus (Ephéméroptère), lacs; 3, larve nymphe d'Arcynopteryx compacta Mc. Lcrl. (A. pyrenaica DEsP) [Plecoptère], lacs plerreux, ruisseaux et sources froides; 4, larve d'Orphnephila (Thaumalea) [Diptère], surfaces hygropétriques; 5, larve de Pericoma cognala EAT. (Diptère), surfaces hygropétriques : 6, larve d'Hermione Pandellet Steo (Diptère), surfaces hygropétriques; 7 et 8, larve et fourreau de Stactobia Mac LAchinani Kim. (S. fuscicornis, auct.) [Trichoptère], surfaces hygropétriques; 9 et 10, larve hygropetrique et imago de Eubria palustris L. (Coléoptère), surfaces hygropétriques; 11 , fourreau de Thremna gallica Mc. Lcal. (Trichoptère), rulsseaux; 12, fourreau de Drusus discolor Ramb. (Trichoptère), ruisseaux. (Bertrand, Collart, Feuerborn, Rousseau, Saunders, Schoenemund). 
des petites cascades et ressauts ; parmi celles-ci, on rencontrera fréquemment les nymphes de Simulium hirtipes, à cocon lâche et très nombreux filaments, et on n'oubliera pas de rechercher, en se portant vers les points où le courant est le plus violent, les cocons argentés de Simulium rupicolum, espèce des Alpes, récemment découverte dans les Pyrénées. Souvent, enfin, en compagnie des Simulies, on rencontre les étranges larves des Blépharocérides, à corps monoliforme, muni de puissantes ventouses ventrales et qu'on ne peut dérocher qu'avec une pression sensible de l'ongle; à côté, souvent, les nymphes noirâtres, nues, avec des branchies cuticulaires en lamelles en avant du thorax.

Mais remontons le cours du Gave et suivons ses rives, ou celles de ses affluents, jusqu'au-dessus même de la limite des forêts, dans les hauts pâturages. Nous retrouverons très sensiblement la même faune, enrichie toutefois non tant en espèces qu'en individus. Nous pouvons encore récolter de petits Plécoptères : Isoperla, Isopteryx, parfois Capnia (en début de saison). On trouvera aussi, surtout dans les petits bras qui sont plutôt des ruisseaux, l'Ephémeroptère Habroleptoides modesta et parmi les Trichoptères, nous ne pouvons manquer de remarquer le fourreau caractéristique du Drusus discolor avec ses brindilles dressées en "ancres", et surtout le fourreau en bonnet phrygien ou coquille d'Ancyle du Thremna gallicum. A cette altitude, ne négligeons pas non plus les Chironomides; ce sont parfois des Tanytarsiens, plutôt des Orthocladiinae ou des Diamesinae, représentés fréquemment par des formes plus ou moins montagnardes dont nous reparlerons plus loin (Diamesa, Eukiefferiella). C'est encore dans le groupe des Diamesinae que l'on range les Heptagia, jadis non connus des Pyrénées, à larves munies de cornes céphaliques et nymphes enveloppées dans des cocons gélatineux collés sur les roches à fleur d'eau, plus exactement même dans la zone éclaboussée ou mouillée par le clapotis de l'eau et la pulsation du courant (splash-line).

Mais ici, si je puis dire, nous commençons à sortir de l'eau ; il existe, en effet, nombre d'êtres vivants dans des conditions intermédiaires : ce sont les hygropétriques et j'ai volontairement omis de parler de toutes les larves ou nymphes que nous aurions pu rencontrer ainsi sur les pierres immergées et surtout au bord de ces grandes et belles cascades si fréquentes le long de nos gaves pyrénéens. Mais pour étudier les milieux hygropétriques, j'ai constaté que l'on pouvait se contenter de se promener le long d'une route. Ainsi, pour prendre un exemple précis, la route thermale près de Barèges, en direction du Tourmalet, entre cette localité et le pont de la Gaubie (environ 3 kilomètres), offre un véritable " échantillonnage "de surfaces hygropétriques et j'y ai récolté la plupart des insectes dont je vais vous entretenir. J'ai mentionné une faune hygropétrique des cascades. Eh bien! à Barèges même, au fond d'une courette sombre et froide, derrière l'abattoir, un petit ruisselet tombe en un long jet, éclaboussant une paroi rocheuse; l'eau coule sur des paquets de mousses tandis que, latéralement, sur plusieurs mètres, la roche est couverte de dépôts glaireux ocrés ou verdâtres où un algologue ferait d'amples récoltes... Plus loin, on rencontre des roches presques nues, balayées par un léger ruissellement; ailleurs s'accrochent des touffes 
d'Algues filamenteuses, voire des lentilles d'eau ; ailleurs encore, le dépôt calcaire affecte l'aspect d'une croûte assez cohérente, tantôt arrosée, tantôt humide, et parfois desséchée. Tout un petit monde vit sur ces surfaces à des degrés d'humidité variable, chaque organisme choisissant selon ses exigences propres, exigences différant d'ailleurs selon l'âge : la larve recherchant souvent plus l'eau que la nymphe, l'imago venant

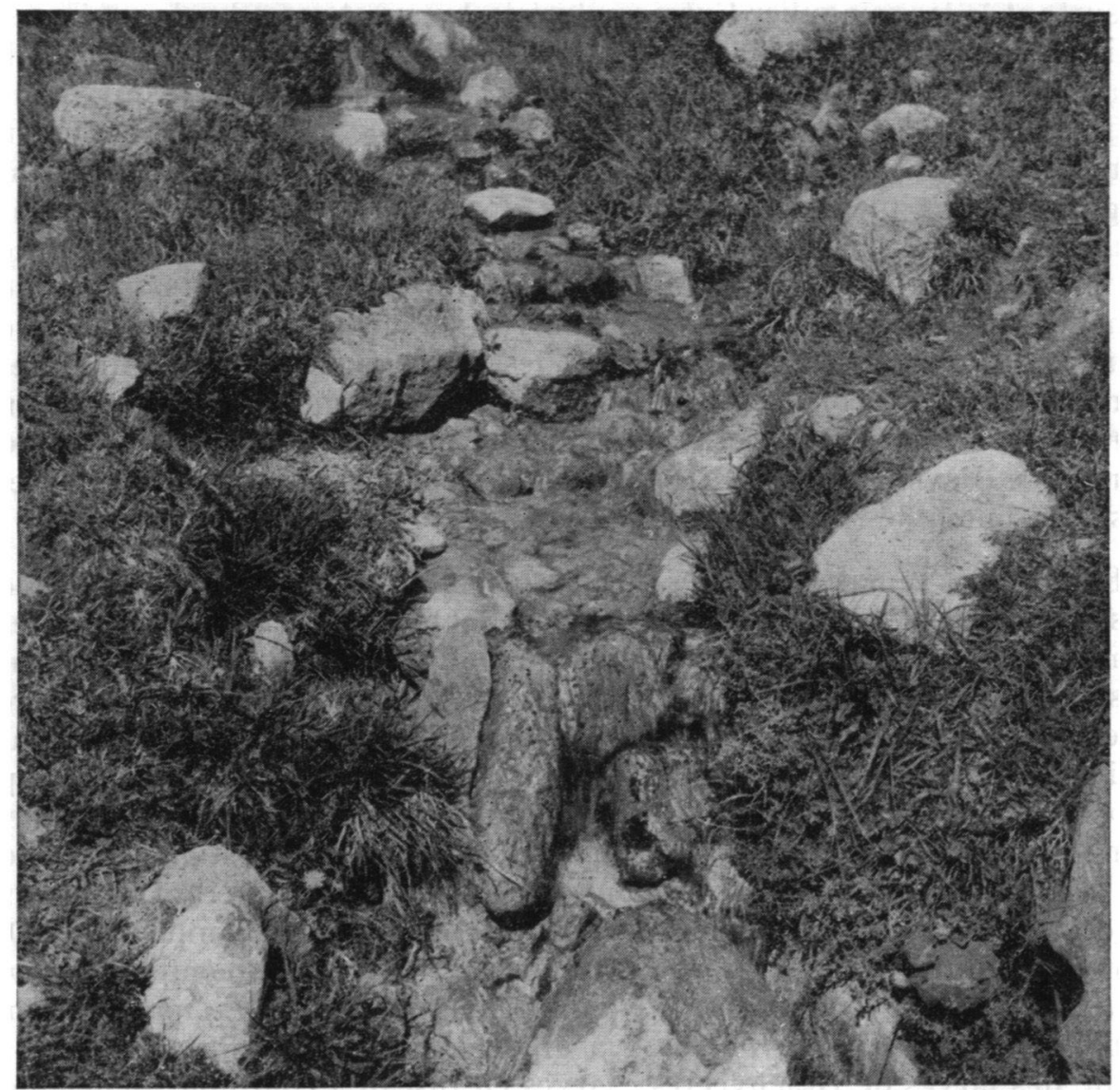

Photo Bertrand

Un ruisseau calcaire à BARÉges. - Station à Lithotangtarsas emarginatus (tư à chironomides).

au plus s'y rafraîchir, sans compter les cadavres qui servent d'aliment aux saprophages. A peu près tous les ordres d'insectes aquatiques se trouvent représentés ici. Plutôt d'ailleurs hygrophiles qu'aquatiques, on verra là des Collemboles (Isotomurus, Tomocerus), des Hémiptères (Salda), quelquefois Gerris et Velia s'aventurent, plus encore les grêles Hydromètres. Il est intéressant d'y retrouver des Plécoptères et Ephémeroptères, ce sont des Nemurides (surtout Nemura) et des Baetis de type alpinus (alpinus, gemellus), rarement des $B$. vernus. Les seconds au moins ne quittent pas le ruissellement vif, faisant front au courant. On trouve encore une faunule particulière de Trichoptères : des Beraea 
à fourreau arqué, des Ptilocolepus à fourreau en feuilles de mousses, quelques larves nues dont les plus caractéristiques sont non tant encore les larves des Psychomyides, cachées dans de longs tubes sinueux collés à la roche, que celles des Stactobia, Hydroptilides à fourreau en tonnelet, groupés en colonies nombreuses. Il n'existe qu'un petit nombre de Coléoptères. On trouve parfois de petits Dryopides (Riolus ou Helmis); plus caractéristiques sont des Hydrophilides des genres Laccobius et Anacaena, et surtout le très singulier Eubria palustris. Larve et nymphe de cet insecte sont réellement étranges, car la première surtout ressemble plus ou moins à un Trilobite; elle possède à la fois deux gros stigmates et des branchies rétractiles et, capable de mener une vie amphibie, se tient généralement hors de l'eau. Les Diptères occupent une place importante dans la faune hygropétrique; bien entendu, nombre de ces insectes, à l'état imaginal recherchent les surfaces hygropétriques; on remarque en particulier de gros Tipules et des Dicranomyia, Diptères des urinoirs, aussi des " mouches" tachées de jaune (Stratiomyides), surtout des Dolichopodides (Liancalus) à reflets métalliques, et les sombres Clinocera (Empidides) portant presque constamment des Acariens rouges (Thyas), encore parmi les Nematocères, les Pericoma qui ressemblent à de minuscules bombyxs (Psychodides ou Mothflies des Anglais), des Orphnephilides, des Chironomides, Cératopogonides.

Parmi les Nematocères, les larves les plus remarquables sont celles des Pericoma (Psychodides) et des Orphnephilides. Les premières ressemblent à de petites chenilles plus ou moins poilues, certaines offrant des poils aplatis où se dépose le calcaire; d'autres - ce sont les plus fréquentes - retenant dans leurs forts poils recourbés un amas de boue qui les dissimule au regard (Pericoma cognata). Les secondes se rapprochent plutôt des larves des Chironomides, mais s'en distinguent bien par la grosse tête munie de bosses, la présence aussi de stigmates; elles se déplacent par de curieux mouvements latéraux différant d'ailleurs de ceux des larves des Culicides Dixa, qui, parfois, les accompagnent. On trouve aussi diverses larves de Chironomides souvent boréoalpins (Gowiniella, Eukiefferiella). Il y a également des larves de Tipula, de Limnophila, surtout de Dicranomyia à gros bourrelets locomoteurs. Il y a, enfin, des larves de Brachycères : larves en fuseau à couronne anale ciliée des Hermione (Stratiomyides), surtout microphages, larves carnassières des Dolichopodides (Liancalus) et des Empidides (Clinocera), blanchâtres, larves également carnassières des Melanochelia (Anthomyides). Mais avant d'en finir avec les larves des Diptères, il est intéressant de signaler la présence parfois de larves de Simulies, notamment Simulium aureum et, au voisinage des cascades, des larves et nymphes de Blépharocérides, ces dernières abondantes, par exemple, dans les ruisselets fort abrupts du profond et sauvage ravin de Malibierne, au cœur des Monts Maudits. Et à ce propos, il convient de remarquer que toute chute d'eau provoquée par une rupture brusque de pente peut arriver à réaliser des conditions plus ou moins hygropétriques; il semble que ce soit au-dessous d'une certaine vitesse de courant et surtout d'une certaine épaisseur de la lame d'eau que s'établit la biocènose hygropétrique. D'ailleurs, dans la nature, 
il existe effectivement des biotopes de passage, tels ces ruisselets de Malibierne. La construction des routes en réalise d'autres; souvent, en effet, on a élevé au niveau des ruisselets une petite murette pour retenir la terre, d'où résulte une cascade en miniature. Dans ces conditions, on observe parfois une formation très remarquable : c'est une mince couche de tuf d'aspect vermiculé, facile à détacher avec la pointe d'un canif, croùte qui est constituée par un dense agrégat de petits tubes calcaires annelés s'achevant en entonnoirs ouverts ou fermés par un opercule percé d'un minuscule orifice, tubes ressemblant à des tubes de Vers annélides. Il s'agit des tubes édifiés par les larves d'un Chironomide Tanytarsien : le Lithotanytarsus, étudié par Thiscemann en Europe centrale et récem-

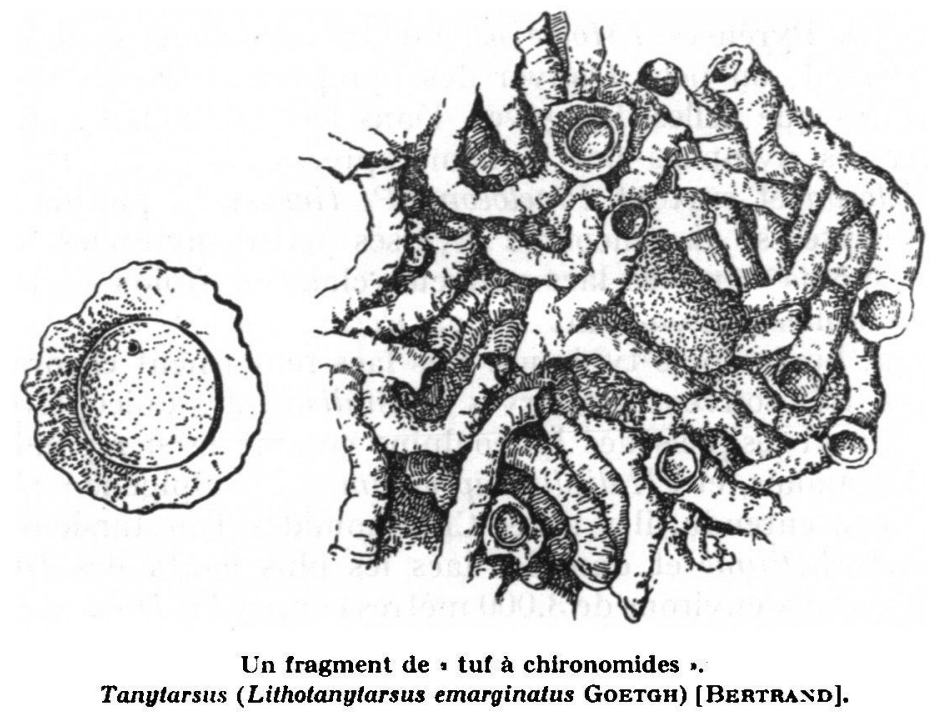

ment découvert dans les Pyrénées. Parfois, ce "tuf à Chironomides" supporte des colonies de Stactobia.

En suivant les routes, nous rencontrons bien entendu de petits ruisseaux, et dans ces ruisseaux nous observerons les nymphes et larves pétricoles des gaves, mais plus abondamment de petites nymphes de Némurides ou d'Ephémeroptères, également des petits Coléoptères, Dryopides, Dytiscides, Hydrophilides, notamment le Dytiscide Deronectes Delarouzei, endémique pyrénéen. Lorsque ces ruisseaux déposent des sels calcaires, on y retrouve, avec une faunule hygropétrique, du tuf à Chironomides.

En montagne comme en plaine, il existe des passages entre les biocénoses lotiques et lénitiques; toutefois, c'est sous la rubrique d'eaux stagnantes que l'on traite habituellement des mares et marécages, couvrant d'assez grands espaces dans les pâturages jusque dans la région subalpine et également des lacs élevés correspondant à des biotopes assez différents. Dans les mares et étangs des bas des vallées, on rencontre souvent une faune banale où peuvent se glisser parfois quelques éléments montagnards (Agabus congener par exemple). Plus haut, dans le pâturage 
élevé, on rencontre des Trichoptères, quelques Chironomides, dont des Tanypodinae, des Culicides, des Hydrophilides de petite taille, des Hélophores dont l'Helophorus glacialis qui se laisse flotter à la surface de l'eau attiédie, et des Dytiscides, aussi bien l'inévitable Agabus guttatus, subalpin, que des Hydroporinae plus ou moins "orophiles". Parfois même, les marais abritent des larves de Dytiscus ou d'Aeschna. Les Hemiptères y sont communs et dans les marettes, même souillées par le bétail, prospèrent les Corises.

Les lacs pyrénéens bien souvent, à première vue, ne paraissent pas très riches; en fait, ils renferment en général un assez petit nombre de formes, encore que la vie animale soit loin d'y être absente ; souvent, ils abritent des têtards de Grenouille ou d'Alyte et dans de nombreux lacs l'Euprocte des Pyrénées (Molge aspera) se dissimule sous les pierres. Vers le déversoir, on peut trouver des nymphes pétricoles qui peuvent être les mêmes que celles des gaves. Dans les lacs mêmes, on a chance de capturer les nymphes des Ephéméroptères : Siphlonurus lacustris, celles des Cloeon et surtout Procloeon (P. Hovassei), parfois de Baetis. Comme Plécoptères, on rencontre diverses petites nymphes, mais aussi bien - au moins dans les lacs pierreux clairs et froids - les grandes et belles nymphes ressemblant à des Perla, mais sans branchies, des Arcynopteryx, boréoalpins typiques. Les lacs renferment encore quelques Trichoptères, très souvent des larves de Sialis et des Coléoptères Hydrocanthares, parmi lesquels des boréoalpins comme le si répandu Agabus Solieri, groënlandais, et l'Haliplus lapponum et l' "alpigène " Hydroporus foveolatus. Bien entendu, il y a des Chironomides Tanytarsiens et Tanypodinae, Orthocladiinae et dans les lacs les plus hauts des Diamesinae, montant jusqu'aux environs de 3.000 mètres comme les Diamesa (D. Steinbocki) de l'ètang glacé du Mont Perdu.

Mais, puisque je me suis aventuré si haut, je ne peux manquer de parler des eaux glaciaires; comme je l'ai dit, les glaciers sont relativement réduits dans les Pyrénées et on ne peut guère faire de comparaison avec les eaux glaciaires des Alpes, explorées par Hubault et Dorier. J'ai toutefois recherché quelle pouvait être la faune des eaux, le plus près possible des glaciers et névés, par exemple près des glaciers du Vignemale et surtout dans le cirque de Gavarnie. C'est dans ce dernier, en partant même des cascades que l'on peut faire les meilleures observations. Sur les pierres mouillées et éclaboussées par des eaux qui paraissent stériles, on voit en nombre de minuscules Collemboles : des Proisostoma, les mêmes d'ailleurs que j'ai rencontrés à près de 3.000 mètres sur les boues glaciaires du col de la Cascade, tout au faîte du cirque; puis apparaissent d'abord des larves de Chironomides d'ailleurs abondantes et bientôt des Rhyacophila, des Leuctra, des Rhitrogena et même des Simulies. Les Chironomides sont des formes boréoalpines ou alpines déjà observées dans les Alpes (Diamesa latitarsis, D. Steinbocki, Eukiefferiella cyanaea).

Je terminerai en disant quelques mots de la faune hypogée des eaux pyrénéennes. Larves, nymphes ou insectes aquatiques peuvent être entraînés dans les eaux souterraines; c'est ainsi que dans les grottes 
de Bétharram, JeAnNel a signalé des nymphes d'Ephemera et d'Habrophlebia, aussi des larves de Psychomyides. Le même auteur fait encore mention de larves d'Hydropsyche dans les grottes espagnoles, également de nymphes de Plécoptères. Des larves de Diptères peuvent vivre aussi dans ces conditions. Enfin, j'ai eu récemment l'occasion d'examiner toute une série d'insectes, larves ou nymphes récoltés par M. ANGelier dans les nappes phréatiques des alluvions de la Tet et surtout du Tech; il y a là des Hydrophilides, Limnebiides, Dryopides, des nymphes d'Ephéméroptères (Ephemerella, Habroleptoides), de Plécoptères (Leuctra), d'Odonates (Gomphines), des larves de Dryopides (Dryops et Helmiinae) et diverses larves des Diptères : Ceratopogonides, Chironomides, Tipulides, Rhagionides (Atherix), Empidides, Tabanides. D'une façon générale, ce sont des insectes d'origine épigée, fréquentant le fond et particulièrement le sable. Toutefois, il est particulièrement intéressant de noter la présence à Prats de Mollo d'une larve de Chironomide boréoalpin : Krenosmittia boreoalpina jamais encore rencontrée en France, en un point où les eaux de surface ont jadis fourni un Turbellarié boréoalpin bien connu : Planaria alpina, recueilli par VANDEL.

Et, pour conclure, je ne puis que répéter une remarque faite au début de cette causerie : tout l'intérêt qu'offrent les Pyrénées tant au point de vue hydrobiologique que biogéographique en général, résulte de la position comme de la structure mêmes de ces montagnes, placées à la limite de deux mondes - un géographe a pu dire qu'au delà commence l'Afrique - baignant dans deux mers, Océan et Méditerranée, assez hautes pour garder le " front de glace " dont parle le poète, assez méridionales pour voir monter la vie jusqu'à leurs cimes dont l'usure rend l'accès dans l'ensemble facile. Et, du point de vue pratique, chacun connaît la richesse économique, notamment piscicole, que représentent ces eaux pyrénéennes d'un si grand attrait. 